NRCPS-HE-2001-4

\title{
The Spectrum of the Loop Transfer Matrix on Finite Lattice
}

\author{
Georgios Daskalakis and George K.Savvidy \\ National Research Center Demokritos, \\ Ag. Paraskevi, GR-15310 Athens, Hellenic Republic
}

\begin{abstract}
We consider the model of random surfaces with extrinsic curvature term embedded into 3d Euclidean lattice $Z^{3}$. On a 3d Euclidean lattice it has equivalent representation in terms of transfer matrix $K\left(Q_{i}, Q_{f}\right)$, which describes the propagation of loops $Q$. We study the spectrum of the transfer matrix $K\left(Q_{i}, Q_{f}\right)$ on finite dimensional lattices. The renormalisation group technique is used to investigate phase structure of the model and its critical behaviour.
\end{abstract}




\section{Introduction}

Various models of random surfaces built out of triangles embedded into continuous space $R^{d}$ and surfaces built out of plaquettes embedded into Euclidean lattice $Z^{d}$ have been considered in the literature [1, 2]. These models are based on area action and suffer the problem of non-scaling behaviour of the string tension and the dominance of branched polymers [3]. Several studies have analyzed the physical effects produced by rigidity of the surface introduced by adding dimension-less extrinsic curvature term to the area action [0]. Comprehensive review of the work in this area up to 1997 can be found in [3].

In this article we shall consider a model of random surfaces solely based on the with extrinsic curvature term embedded into 3d Euclidean lattice $Z^{3}$ [5]. The corresponding transfer matrix has the form [6]

$$
K\left(Q_{1}, Q_{2}\right)=\exp \left\{-\beta\left[k\left(Q_{1}\right)+2 l\left(Q_{1} \triangle Q_{2}\right)+k\left(Q_{2}\right)\right]\right\},
$$

where $Q_{1}$ and $Q_{2}$ are closed polygon-loops on a two-dimensional lattice, $k(Q)$ is the curvature and $l(Q)$ is the length of the polygon-loop $Q$. This transfer matrix describes the propagation of the initial loop $Q_{1}$ to the final loop $Q_{2}$.

The spectrum of the transfer matrix which depends only on symmetric difference of initial and final loops $Q_{1} \triangle Q_{2}$

$$
\tilde{K}\left(Q_{1}, Q_{2}\right)=\exp \left\{-2 \beta l\left(Q_{1} \triangle Q_{2}\right)\right\},
$$

has been evaluated analytically in terms of correlation functions of the $2 \mathrm{~d}$ Ising model in [8].

Our aim in this article is to consider the full transfer matrix (1) which depends not only on symmetric difference of loops $Q_{1} \triangle Q_{2}$, but also on individual curvature terms $k\left(Q_{1}\right)+k\left(Q_{2}\right)$. Because analytical solution of this problem is not known yet, we shall consider numerical evaluation of the transfer matrix on finite lattices in order to gain new insight into spectral properties of the transfer matrix in general case and critical properties of the model.

In the next sections we shall numerically compute the spectrum of transfer matrix on finite Euclidean lattices $T^{2}$ of the sizes $N \star M$, where $N, M=1,2,3,4$. Number of loops on these lattices $\gamma=2^{N M}$ will be 2,4,16,64,512,4096, 65536 and transfer matrices would be of the size $\gamma \times \gamma=2^{N M} \times 2^{N M}$.

\section{Basic Formulae}

The partition function is defined as [6]

$$
Z(\beta)=\sum_{\left\{Q_{1}, Q_{2}, \ldots, Q_{\tau}\right\}} K_{\beta}\left(Q_{1}, Q_{2}\right) \cdots K_{\beta}\left(Q_{\tau}, Q_{1}\right)=\operatorname{tr} K_{\beta}^{\tau},
$$

where $K_{\beta}\left(Q_{1}, Q_{2}\right)$ is the transfer matrix of size $\gamma \times \gamma$, defined above (11). $Q_{1}$ and $Q_{2}$ are closed polygon-loops on a two-dimensional lattice $T^{2}$ of size $N \times M$ and $\gamma=2^{N M}$ is the total number of polygon-loops on a lattice $T^{2}$. The transfer matrix (11) can be viewed

\footnotetext{
${ }^{1}$ We shall use the word "loop" for the "polygon-loop".
} 
as describing the propagation amplitude of the polygon-loop $Q_{1}$ at time $\tau$ to another polygon-loop $Q_{2}$ at the time $\tau+1$.

The functional $k(Q)$ is the total curvature of the polygon-loop $Q$ which is equal to the number of corners of the polygon ( the vertices with self-intersection are not counted!) and $l(Q)$ is the length of $Q$ which is equal to the number of its links. The length functional $l\left(Q_{1} \triangle Q_{2}\right)$ is defined as [6]

$$
l\left(Q_{i}\right)+l\left(Q_{i+1}\right)-2 \cdot l\left(Q_{i} \cap Q_{i+1}\right)=l\left(Q_{i} \triangle Q_{i+1}\right),
$$

where the polygon-loop $Q_{1} \triangle Q_{2} \equiv Q_{1} \cup Q_{2} \backslash Q_{1} \cap Q_{2}$ is a union of links $Q_{1} \cup Q_{2}$ without common links $Q_{1} \cap Q_{2}$. The operation $\triangle$ maps two polygon-loops $Q_{1}$ and $Q_{2}$ into a polygon-loop $Q=Q_{1} \triangle Q_{2}$.

The eigenvalues of the transfer matrix $K_{\beta}\left(Q_{1}, Q_{2}\right)$ define all statistical properties of the system and can be found as a solution of the following integral equation in the loop space $\Pi[$ [ $]$

$$
\sum_{\left\{Q_{2}\right\}} K_{\beta}\left(Q_{1}, Q_{2}\right) \Psi\left(Q_{2}\right)=\Lambda(\beta) \Psi\left(Q_{1}\right),
$$

where $\Psi(Q)$ is a function on the loop space. The eigenvalues define the partition function (3)

$$
Z_{N M}^{3 d}(\beta)=\Lambda_{0}^{\tau}(\beta, N M)+\ldots+\Lambda_{\gamma-1}^{\tau}(\beta, N M),
$$

and in the thermodynamical limit the free energy is equal to

$$
-\beta f_{3 d}(\beta)=\lim _{\tau N M \rightarrow \infty} \frac{1}{\tau N M} \ln Z^{3 d}(\beta)=\lim _{N, M \rightarrow \infty} \frac{1}{N M} \ln \Lambda_{0}(\beta, M N),
$$

where $\Lambda_{0}(\beta, N M)$ is the largest eigenvalue defined at temperature $\beta$ for the lattice of the size $N \times M$. The correlation length is defined by the ratio of eigenvalues $\Lambda_{1}(\beta, N M)$ and $\Lambda_{0}(\beta, N M)$ as

$$
\xi_{N M}(\beta)=-1 / \ln \left[\Lambda_{1}(\beta, N M) / \Lambda_{0}(\beta, N M)\right],
$$

and grows if the eigenvalue $\Lambda_{1}$ approaches to the eigenvalue $\Lambda_{0}$ at some critical temperature $\beta_{c}$. By the Frobenius-Perron theorem $\Lambda_{0}(\beta)$ is simple and $\Lambda_{0}(\beta)>\Lambda_{1}(\beta) \geq \Lambda_{2}(\beta) \geq$

Because we can analyze numerically only finite-dimensional matrices we shall use renormalisation group approach to critical phenomena [9] in order to extract critical indexes. The renormalisation group equation

$$
\bar{\beta}=\bar{\beta}(\beta)
$$

is defined through the correlation function

$$
\frac{\xi_{N M}(\beta)}{N+M}=\frac{\xi_{\bar{N} \bar{M}}(\bar{\beta})}{\bar{N}+\bar{M}}, \quad N+M>\bar{N}+\bar{M}
$$

The critical point $\beta_{c}$ is a fixed point $\beta^{*}$ of the renormalisation group equation (8)

$$
\beta^{*}=\bar{\beta}\left(\beta^{*}\right) .
$$

The renormalisation group equation (8) can be expanded near fixed point $\beta^{*}$ into the series of the following form

$$
\bar{\beta}(\beta)-\beta^{*}=g_{1}\left(\beta-\beta^{*}\right)+g_{2}\left(\beta-\beta^{*}\right)^{2}+\ldots
$$




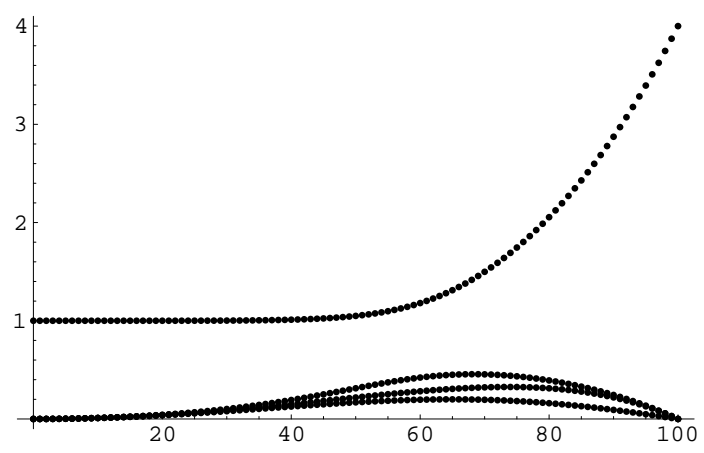

Figure 1: The typical behaviour of the eigenvalues. These are eigenvalues of the transfer matrix $\mathrm{K}$ as a function of $\omega=e^{-4 \beta}$ for the size $4 \times 4$.

and the critical index $\nu$ is equal to

$$
\nu=\frac{\ln [(N+M) /(\bar{N}+\bar{M})]}{\ln g_{1}} .
$$

We shall use these formulas to define fixed point and the critical indexes.

In the next sections we shall explicitly construct transfer matrices on finite Euclidean lattices $T^{2}$ of the sizes $N \star M$, where $N, M=1,2,3,4$. Number of loops on these lattices $\gamma=2^{N M}$ will be $2,4,16,64,512,4096,65536$ and transfer matrices would be of the size $\gamma \times \gamma=2^{N M} \times 2^{N M}$.

\section{General properties of the spectrum}

We shall fix the boundary spins on a lattice $T^{2}$, let us say, in up direction. The convention is that we shall not count the boundary spins, thus if the lattice has the size $2 \times 3$ then it has six internal spins 2. In this setting when all spins are in up direction then in the dual picture we shall have just an empty loop. When one spin inside lattice is turned down, then in the dual picture we shall have simply one loop consisting of four bonds, a box-loop, and so on.

Let us begin from the simplest lattice of the size $1 \times 1$ and then move to more complicated ones in order to understand general properties of the spectrum. For this lattice which has just one spin we have empty loop $\emptyset$ and one box-loop $\square$. The transfer matrices $K$ and $\tilde{K}$ describing transitions between these two loops can be computed by using definition (1) and (2)

$$
K=\left(\begin{array}{lr}
1 & e^{-12 \beta} \\
e^{-12 \beta} & e^{-8 \beta}
\end{array}\right), \quad \tilde{K}=\left(\begin{array}{lr}
1 & e^{-8 \beta} \\
e^{-8 \beta} & 1
\end{array}\right),
$$

and then it is easy to find its eigenvalues:

$$
2 \Lambda_{0,1}=1+e^{-8 \beta} \pm \sqrt{1+4 e^{-24 \beta}+e^{-16 \beta}-2 e^{-8 \beta}}, \quad \tilde{\Lambda}_{0,1}=1 \pm e^{-8 \beta} .
$$

For the lattice of the size $1 \times 2$ we have four loops: the empty loop, two one-box loops

\footnotetext{
${ }^{2}$ And ten boundary spins which we shall not count.
} 


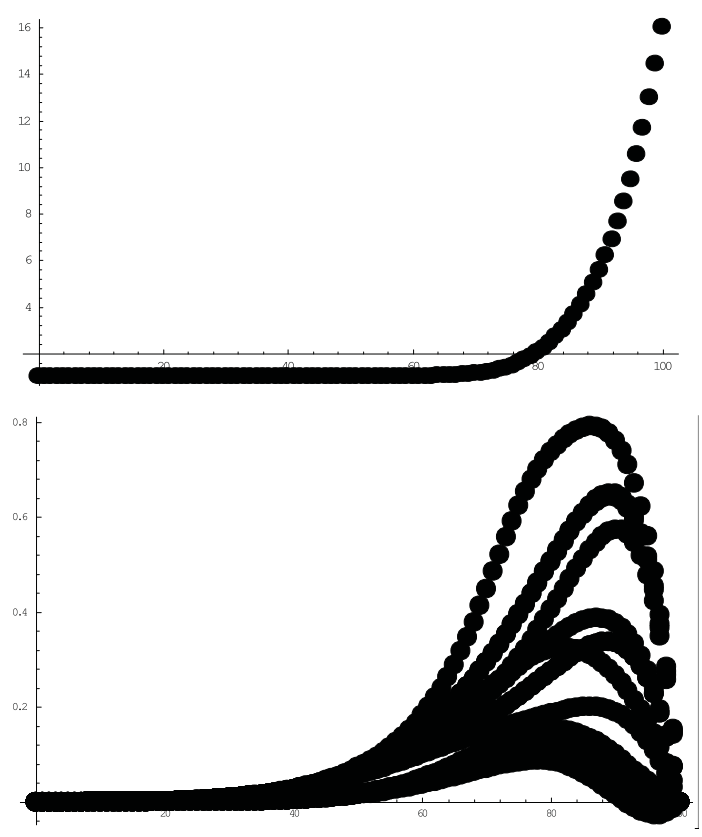

Figure 2: The spectrum of the transfer matrices $K$ of the size $16 \times 16$ as a function of $\omega=e^{-2 \beta}$. The qualitative character of the spectrum is the same as in Figure 1, but now we can see that at high temperatures some of the eigenvalues become negative.

and one two-box loop. The transfer matrices are therefore four by four:

$$
K=\left(\begin{array}{llll}
1 & e^{-12 \beta} & e^{-12 \beta} & e^{-16 \beta} \\
e^{-12 \beta} & e^{-8 \beta} & e^{-20 \beta} & e^{-16 \beta} \\
e^{-12 \beta} & e^{-20 \beta} & e^{-8 \beta} & e^{-16 \beta} \\
e^{-16 \beta} & e^{-16 \beta} & e^{-16 \beta} & e^{-8 \beta}
\end{array}\right), \quad \tilde{K}=\left(\begin{array}{llll}
1 & e^{-8 \beta} & e^{-8 \beta} & e^{-12 \beta} \\
e^{-8 \beta} & 1 & e^{-12 \beta} & e^{-8 \beta} \\
e^{-8 \beta} & e^{-12 \beta} & 1 & e^{-8 \beta} \\
e^{-12 \beta} & e^{-8 \beta} & e^{-8 \beta} & 1
\end{array}\right)
$$

The eigenvalues of the matrix $\tilde{K}$ have been found in 8

$$
\tilde{\Lambda}_{0}=1+2 e^{-8 \beta}+e^{-12 \beta}, \quad \tilde{\Lambda}_{1,2}=1-e^{-12 \beta}, \quad \tilde{\Lambda}_{3}=1-2 e^{-8 \beta}+e^{-12 \beta},
$$

and for the matrix $K$ we have found eigenvalues on a computer (see Figure 11). In both cases all eigenvalues are positive. As the last example of explicitly depicted transfer matrix 
we shall present it for the lattice $2 \times 2$. It has sixteen loops and is of the size $16 \times 16$

$$
K=\left(\begin{array}{llllllllllllllll}
0 & 6 & 6 & 6 & 6 & 8 & 8 & 8 & 8 & 11 & 11 & 11 & 11 & 11 & 11 & 10 \\
6 & 4 & 10 & 12 & 10 & 8 & 12 & 8 & 12 & 9 & 13 & 11 & 13 & 11 & 13 & 12 \\
6 & 10 & 4 & 10 & 12 & 8 & 12 & 12 & 8 & 13 & 9 & 13 & 11 & 13 & 11 & 12 \\
6 & 12 & 10 & 4 & 10 & 12 & 8 & 12 & 8 & 9 & 13 & 11 & 13 & 11 & 13 & 12 \\
6 & 10 & 12 & 10 & 4 & 12 & 8 & 8 & 12 & 13 & 9 & 13 & 11 & 13 & 11 & 12 \\
8 & 8 & 8 & 12 & 12 & 4 & 12 & 12 & 12 & 11 & 11 & 9 & 13 & 13 & 9 & 10 \\
8 & 12 & 12 & 8 & 8 & 12 & 4 & 12 & 12 & 11 & 11 & 13 & 9 & 9 & 13 & 10 \\
8 & 8 & 12 & 12 & 8 & 12 & 12 & 4 & 12 & 9 & 11 & 13 & 13 & 9 & 9 & 10 \\
8 & 12 & 8 & 8 & 12 & 12 & 12 & 12 & 4 & 11 & 11 & 9 & 9 & 13 & 13 & 10 \\
11 & 9 & 13 & 9 & 13 & 11 & 11 & 9 & 11 & 6 & 14 & 10 & 14 & 10 & 14 & 9 \\
11 & 13 & 9 & 13 & 9 & 11 & 11 & 11 & 11 & 14 & 6 & 14 & 10 & 14 & 10 & 9 \\
11 & 11 & 13 & 11 & 13 & 9 & 13 & 13 & 9 & 10 & 14 & 6 & 12 & 14 & 12 & 9 \\
11 & 13 & 11 & 13 & 11 & 13 & 9 & 13 & 9 & 14 & 10 & 12 & 6 & 12 & 14 & 9 \\
11 & 11 & 13 & 11 & 13 & 13 & 9 & 9 & 13 & 10 & 14 & 14 & 12 & 6 & 12 & 9 \\
11 & 13 & 11 & 13 & 11 & 9 & 13 & 9 & 13 & 14 & 10 & 12 & 14 & 12 & 6 & 9 \\
10 & 12 & 12 & 12 & 12 & 10 & 10 & 10 & 10 & 9 & 9 & 9 & 9 & 9 & 9 & 4
\end{array}\right)
$$

where we show only the exponents. The eigenvalues of the matrix $\tilde{K}$ have been found in [8]

$$
\begin{array}{ll}
\tilde{\Lambda}_{0}=1+4 e^{-8 \beta}+6 e^{-12 \beta}+5 e^{-16 \beta}, & \tilde{\Lambda}_{1,2,3,4}=1+2 e^{-8 \beta}-3 e^{-16 \beta}, \\
\tilde{\Lambda}_{5,6,7,8}=1-2 e^{-12 \beta}+2 e^{-16 \beta}, & \tilde{\Lambda}_{9,10}=1-2 e^{-12 \beta}+e^{-16 \beta}, \\
\tilde{\Lambda}_{11,12,13,14}=1-2 e^{-8 \beta}+e^{-16 \beta}, & \tilde{\Lambda}_{15}=1-4 e^{-8 \beta}+6 e^{-12 \beta}-3 e^{-16 \beta} .
\end{array}
$$

The eigenvalues of matrix $K$ are shown on Figure 2. The general properties of the spectrum at low and high temperatures can be easily understood. At $\beta \rightarrow 0$ the eigenvalues are :

$$
\Lambda_{0}=2^{N M}, \quad \Lambda_{1}=\ldots \ldots=\Lambda_{2^{N M}-1}=0
$$

and when $\beta \rightarrow \infty$ they are :

$$
\Lambda_{0}=1, \quad \Lambda_{1}=\ldots . .=\Lambda_{2^{N M}-1}=0 .
$$

These properties of the eigenvalues at the ends of the spectrum can be seen on Figures 1 and 2. These Figures also allow to see the behaviour of the spectrum at the intermediary temperatures, the largest eigenvalue $\Lambda_{0}$ and the second one $\Lambda_{1}$ approach to each other. The rest of the eigenvalues follow the behaviour of the $\Lambda_{1}$. As we will see minimal distance between eigenvalue $\Lambda_{0}$ and $\Lambda_{1}$ decreases when the size of the lattice increases signaling the possibility of a phase transition.

\section{Numerical calculation of eigenvalues $\Lambda_{0}$ and $\Lambda_{1}$}

To generate the transfer matrices of the larger size we have developed a special fast algorithm to create polygon-loops $Q$ and then to compute the elements of the transfer matrix $K$. This part of the program is working fast enough to generate matrices of the size 65536 and higher. To compute eigenvalues $\Lambda_{0}$ and $\Lambda_{1}$ we have used standard technique, that is acting by the matrix $K$ on an arbitrary vector $\Psi(Q)$ many, $m$, times. In the limit 


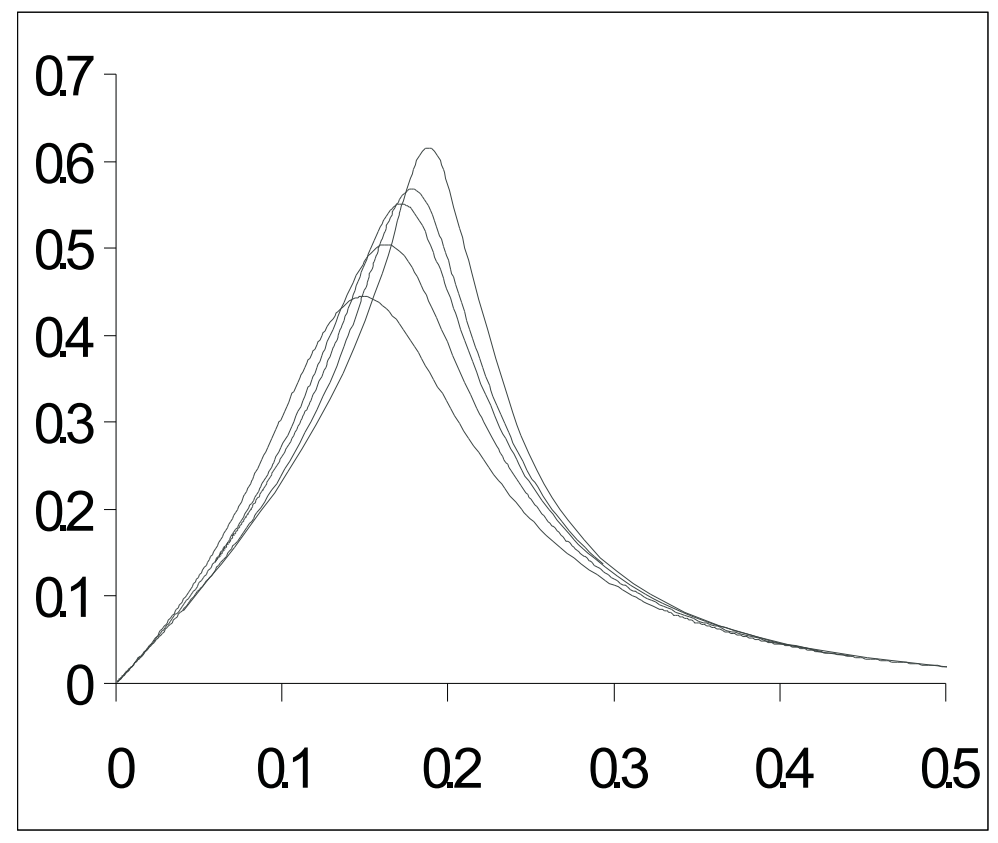

Figure 3: The ratio $\Lambda_{1} / \Lambda_{0}$ as a function of $\beta$ for the matrices of sizes $16 \times 16,64 \times$ $64,256 \times 256,512 \times 512,4096 \times 4096$.

$m \rightarrow \infty$ it converges to $\Psi_{0}(Q)$. In the same manner, acting on the orthogonal vector, one can get $\Psi_{1}(Q)$ and therefore corresponding eigenvalues. The real problem to handle higher size matrices is mostly connected with the memory of the computer.

The ratio of the eigenvalues $\Lambda_{0} / \Lambda_{1}$ is shown on Figure 3. This ratio increases with the size of the matrix $K$ and reaches the value 0.6 for the matrix of the size $4096 \times 4096$. To find fixed point $\beta^{*}$ of the renormalization group equation we have constructed the curves $\xi_{N M}(\beta) /(N+M)$ for different lattices $N * M$. As one can see on Figure 4 there are two fixed points at $\beta_{1}^{*} \simeq 0.17$ and at $\beta_{2}^{*} \simeq 0.21$. At the stable fixed point $\beta_{2}^{*} \simeq 0.21$ we have computed the value $g_{1} \simeq 1.99$ and the critical index $\nu \simeq 0.59$. This should be compared with the results obtained in [13] by low temperature expansion and by MonteCarlo simulation of the corresponding system in [14, 15]. Our value is of the same order of magnitude. To confirm these results one should go to high-dimensional matrices.

It is also interesting to see the behaviour of eigenfunctions. The eigenfunctions $\Psi(Q)$ for the matrix $\tilde{K}(\beta)$ are already known and are independent of the temperature [8]. This is because transfer matrices $\tilde{K}(\beta)$ commute with each other at different temperatures and the system is integrable [8]. This is a general property of integrable systems [7, 10, 11, 12]. The situation with the full transfer matrices $K(\beta)$ is not known, therefore we should check if the eigenfunctions are temperature dependent. For that reason we shall compute the scalar product

$$
\left(\Psi_{\Lambda}(Q)\left(\beta^{\prime}\right) \star \Psi_{\Lambda}(Q)(\beta)\right)
$$

between eigenfunction $\Psi_{\Lambda}(Q)(\beta)$ at different temperatures $\beta^{\prime}$ and $\beta$ in order to see if the scalar product depends on temperature. For the first eigenvalue $\Lambda_{0}$ and $\beta^{\prime}=0$ the result of the calculations is shown on Figure 5. It is clearly seen that the scalar product is temperature dependent and that it drastically changes near the critical points. This result drives us to the conclusion that full transfer matrix represents a non-integrable system. 


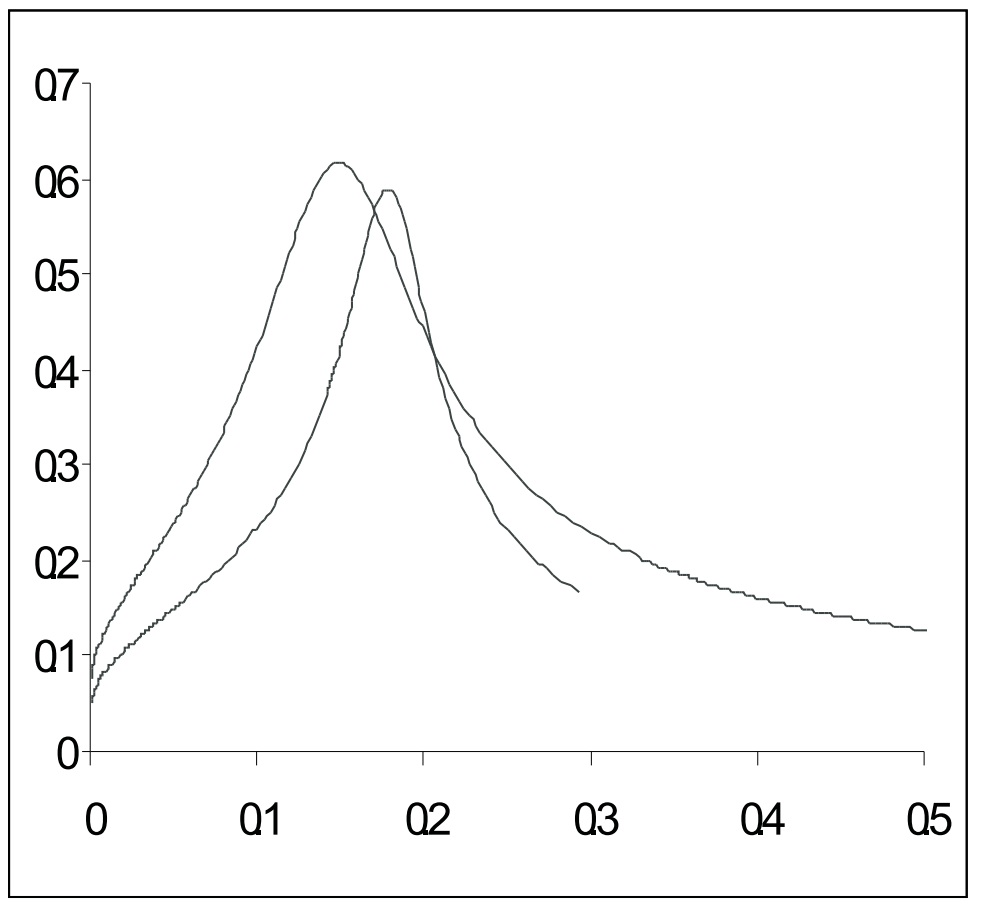

Figure 4: The ratio $\xi_{N M}(\beta) /(N+M)$ as a function of $\beta$ for the matrices of sizes $16 \times 16$ and $512 \times 512$. There are two fixed points at $\beta_{1}^{*} \simeq 0.17$ and at $\beta_{2}^{*} \simeq 0.21$.

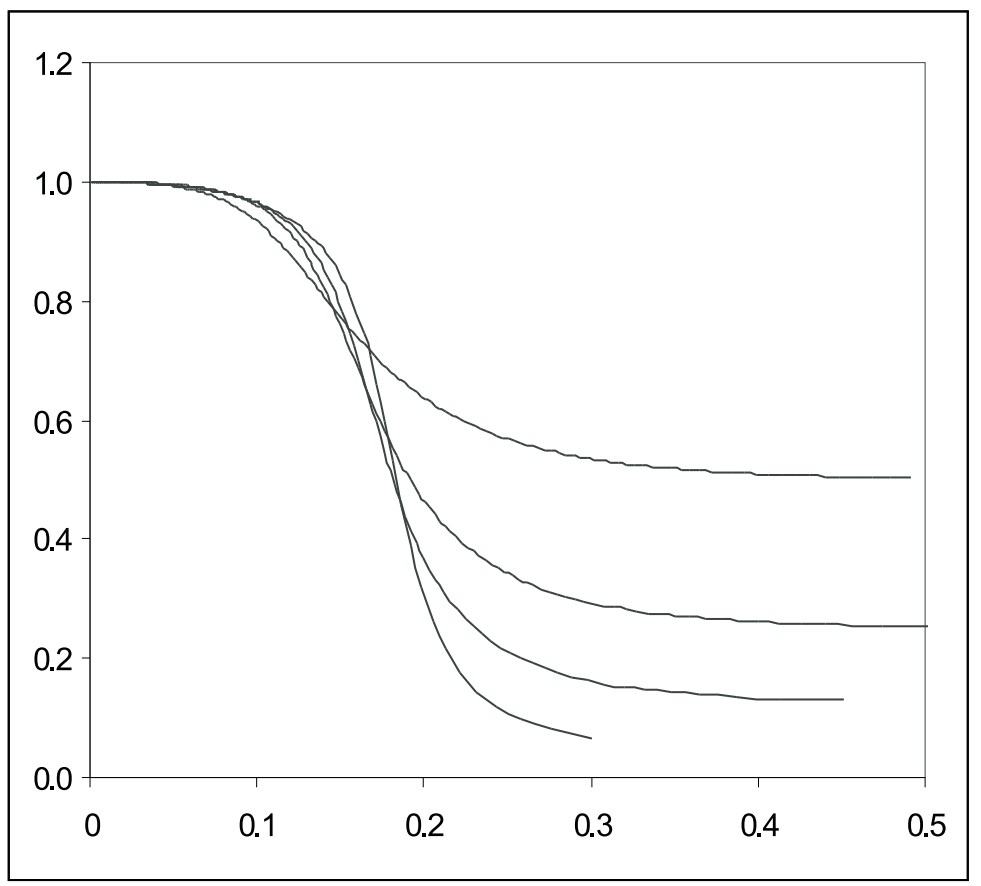

Figure 5: Temperature dependents of the scalar product $\left\{\Psi_{0}(Q)(0) \star \Psi_{0}(Q)(\beta)\right\}$ for the matrices of the sizes $16 \times 16,64 \times 16,512 \times 512,4096 \times 4096$. 
We would like to thank Thordur Jonsson for stimulating discussions and A.Peppas for his collaboration in early stages of this work and calculation of eigenvalues for low dimensional matrices. This work was supported in part by the EEC Grant no. HPRNCT-1999-00161 and the Grant from Ministry of Research and Technology: "Bilateral Cooperation between Hellenic Republic and Georgia".

\section{References}

[1] D.Weingarten. Nucl.Phys.B210 (1982) 229

A.Maritan and C.Omero. Phys.Lett. B109 (1982) 51

T.Sterling and J.Greensite. Phys.Lett. B121 (1983) 345

B.Durhuus,J.Fröhlich and T.Jonsson. Nucl.Phys.B225 (1983) 183

J.Ambjørn,B.Durhuus,J.Fröhlich and T.Jonsson. Nucl.Phys.B290 (1987) 480

T.Hofsäss and H.Kleinert. Phys.Lett. A102 (1984) 420

M.Karowski and H.J.Thun. Phys.Rev.Lett. 54 (1985) 2556

F.David. Europhys.Lett. 9 (1989) 575

[2] D.Gross. Phys.Lett. B138 (1984) 185

V.A.Kazakov. Phys.Lett. B150 (1985) 282

F.David. Nucl.Phys. B257 (1985) 45

J.Ambjørn, B.Durhuus and J.Fröhlich. Nucl.Phys. B257 (1985) 433

V.A.Kazakov, I.K.Kostov and A.A.Migdal. Phys.Lett. B157 (1985) 295

[3] J.Ambjorn, B.Durhuus and T.Jonsson. Quantum geometry. Cambridge Monographs on Mathematical Physics. Cambridge 1998; J.Phys.A 21 (1988) 981

[4] W.Helfrich. Z.Naturforsch. C28 (1973) 693; J.Phys.(Paris) 46 (1985) 1263

L.Peliti and S.Leibler. Phys.Rev.Lett. 54 (1985) 1690

A.Polykov. Nucl.Phys.B268 (1986) 406

D.Forster. Phys.Lett. 114A (1986) 115

H.Kleinert. Phys.Lett. 174B (1986) 335

T.L.Curtright and et.al. Phys.Rev.Lett. 57 (1986)799; Phys.Rev. D34 (1986) 3811

F.David. Europhys.Lett. 2 (1986) 577

P.O.Mazur and V.P.Nair. Nucl.Phys. B284 (1987) 146

E.Braaten and C.K.Zachos. Pys.Rev. D35 (1987) 1512

E.Braaten, R.D.Pisarski and S.M.Tye. Phys.Rev.Lett. 58 (1987) 93

P.Olesen and S.K.Yang. Nucl.Phys. B283 (1987) 73

R.D.Pisarski. Phys.Rev.Lett. 58 (1987) 1300

[5] R.V. Ambartzumian, G.K. Savvidy , K.G. Savvidy

and G.S. Sukiasian. Phys. Lett. B275 (1992) 99

G.K. Savvidy and K.G. Savvidy. Mod.Phys.Lett. A8 (1993) 2963

G.K. Savvidy and K.G. Savvidy. Int. J. Mod. Phys. A8 (1993) 3993

G.K.Savvidy and F.J.Wegner. Nucl.Phys.B413(1994)605

G.K. Savvidy and K.G. Savvidy. Phys.Lett. B324 (1994) 72 
[6] G.K. Savvidy and K.G. Savvidy. Phys.Lett. B337 (1994) 333;

Mod.Phys.Lett. A11 (1996) 1379.

G.K. Savvidy, K.G. Savvidy and P.K.Savvidy Phys.Lett. A221 (1996) 233

[7] H.A.Kramers and G.H.Wannier. Phys.Rev. 60 (1941) 252

L.Onsager. Phys.Rev. 65 (1944) 117

M.Kac and J.C.Ward. Phys.Rev. 88 (1952) 1332

C.A.Hurst and H.S.Green. J.Chem.Phys. 33 (1960) 1059

[8] T.Jonsson and G.K.Savvidy. Phys.Lett.B449 (1999) 254

T.Jonsson and G.K.Savvidy. Nucl.Phys. B575 (2000) 661

G.K.Savvidy. JHEP 0009 (2000) 044

[9] M.P.Nightingale. Physica 83A (1976) 561

[10] R.J.Baxter, Exactly solved models in statistical mechanics. Academic Press, London 1982

[11] A.B. Zamolodchikov. Commun.Math.Phys.79 (1981) 489

V.V.Bazhanov and R.J.Baxter. J.Stat.Phys. 69(1992) 453

[12] A.Polyakov. Gauge fields and String. (Harwood Academic Publishers, 1987)

E.Fradkin, M.Srednicky and L.Susskind. Phys.Rev. D21 (1980) 2885

C.Itzykson. Nucl.Phys. B210 (1982) 477

A.Casher, D.Foerster and P.Windey. Nucl.Phys. B251 (1985) 29

[13] R. Pietig and F.J. Wegner. Nucl.Phys. B466 (1996) 513;

Nucl.Phys.B525 (1998) 549

[14] G.K.Bathas et.al. Mod.Phys.Lett. A10 (1995) 2695

D.Johnson and R.K.P.C.Malmini, Phys.Lett. B378 (1996) 87

M.Baig, D.Espriu, D.Johnson and R.K.P.C.Malmini, J.Phys. A30 (1997) 407; J.Phys. A30 (1997) 7695

G.Koutsoumbas et.al. Phys.Lett. B410 (1997) 241

[15] A.Cappi, P.Colangelo, G.Gonella and A.Maritan, Nucl. Phys. B370 (1992) 659

G.Gonnella, S.Lise and A.Maritan, Europhys. Lett. 32 (1995) 735

E.N.M.Cirillo and G.Gonella. J.Phys.A: Math.Gen.28 (1995) 867 